\title{
Las redes sociales digitales: una valoración socioeducativa. Revisión sistemática
}

\section{Social networks: a socio-educational vision. Systematic review}

\author{
(iD) Dieter Reynaldo Fuentes Cancell \\ Universidad de las Ciencias Informáticas (Cuba) \\ (iD) Odiel Estrada Molina \\ Universidad de las Ciencias Informáticas (Cuba) \\ (iD) Nilda Delgado Yanes \\ Universidad de las Ciencias Informáticas (Cuba)
}

\begin{abstract}
Resumen
La futura y ya actual revolución industrial 4.0 demanda la introducción de una transformación digital en la educación superior contribuyendo a la formación de profesionales competentes, por lo cual, en las pedagogías y tecnologías emergentes se evidencian un incremento de experiencias didácticas en el uso de las redes sociales digitales. En esta investigación se propone a partir de una revisión sistemática cuáles son las tendencias actuales del uso de las redes sociales digitales con fines educativos. Se empleó el protocolo PRISMA y se analizaron 79 trabajos presentes en la base de datos Scopus. En la revisión sistemática, se responden las siguientes preguntas: ¿Qué tipo de diseños predominan en la literatura científica? ¿Cuáles son los pares de variables dependiente - independiente? y ¿Cuáles son las tendencias actuales del uso de las redes sociales digitales con fines educativos? Como resultado de la investigación se reafirma Facebook como la red social más empleada por los educadores y la necesidad del uso integrado y variado de estas redes. Se concluye con la defensa de las siguientes tendencias: políticas de comunicación institucional universitaria desde los niveles macro, meso y micro del currículo; el desarrollo de la creatividad, la convergencia cultural y la diversificación mediática; la innovación educativa; la cultura mediática y la identidad digital académica.
\end{abstract}

\section{Abstract}

The future and already current industrial revolution 4.0 demand the introduction of a digital transformation in the higher education contributing to the formation of competent professionals, for which, they are evidenced in the pedagogies and emergent technologies, an increase of didactic experiences in the use of the digital social networks. In this research a systematic review to identify current trends in the use of online social networks for educational purposes are performed. PRISMA protocol was used and analyzed 79 studies present in the database Scopus. In the systematic review, the following questions are answered: What types of designs predominate in the scientific literature? What is the dependentindependent pairs of variables? And What are the current trends in the use of digital social networks for educational purposes? As a result of this research, Facebook is reaffirmed as the social network most used by educators and the need for the integrated and varied use of these networks. It concludes with the defense of the following trends: university institutional communication policies from the curricular levels; the development of creativity, cultural convergence and media diversification; educational innovation; media culture and academic digital identity.

\section{Palabras clave / Keywords}

Aprendizaje; comunicación interactiva; educación; educación superior; estudio bibliográfico; formación; interacción social; redes sociales.

Learning; interactive communication; education; higher education; literature reviews; teaching; intergroup relations; social networks. 


\section{Introducción}

Las redes sociales digitales (RSD) enriquecen la práctica pedagógica de la educación (Núñez, 2018) a partir de las potencialidades tecnológicas y diversidad de los estilos de aprendizajes. Conocer el cómo influyen en el aprendizaje y su dinámica en la formación del profesional, son eslabones claves para la educación mediática y cultural. Por tal motivo un profesional es valorado por el dominio de sus competencias profesionales pero también, por su capacidad de analizar, interpretar, valorar y decidir en el mundo digital de las publicaciones mediáticas actuales y de lograr una adecuada apreciación, respeto, valoración artística y cultural de su entorno personal y social.

Alvídrez y Franco-Rodríguez (2016) identificaron las potencialidades de las RSD en la participación cívica. De manera similar, diversos estudios (Manca; 2019; Niu, 2019; Thai, Sheeran \& Cummings, 2019; Baker-Doyle \& Yoon, 2020) presentan resultados en la aplicación de estas redes en la educación cívica, promoviendo así la educación formal e informal. En esta dirección, Cabalin y Antezana (2016) defienden la orientación ideológica desde las RSD.

En investigaciones educativas analizadas (indexadas en Scopus) referentes al uso de las RSD se evidencia un marcado interés fundamentalmente en las siguientes variables: competencia cultural, educación de género, trabajo colaborativo, comunidades de aprendizaje, motivación, rendimiento académico, e-learning, b-learning y el empoderamiento del alumnado (Chang, Guo \& Lin, 2017; Verdú, Contreras-Pulido \& GuzmánFranco, 2019; Hermann-Acosta, Apolo \& Molano-Camargo, 2019; Izquierdo-Iranzo \& Gallardo-Echenique, 2020). Lo anterior es muestra de un creciente beneficio de emplear estas redes como complemento en el empleo de las tecnologías emergentes. Si bien diversas investigaciones experimentales han estudiado el efecto del empleo de las RSD en el aprendizaje del estudiantado, se carecen de estudios de tipo revisiones sistemáticas, mapeos sistemáticos o de revisión bibliográfica que, dentro de sus límites metodológicos, exploren y sistematicen los principales resultados obtenidos.

En el último quinquenio diversos estudios teóricos se han publicado e indexado en Scopus, argumentando tendencias de las RSD en la educación, destacándose el aprendizaje colaborativo, la interactividad y el desarrollo de competencias digitales, comunicativas y lingüísticas (Mnkandla \& Minnaar, 2017; Zachos, Paraskevopoulou-Kollia \& Anagnostopoulos, 2018; Scott \& Goode, 2020). Para lo cual se aplican metodologías y procedimientos basados en los diversos estilos de aprendizaje y la multiculturalidad de los estudiantes. Sin embargo, aunque se argumentan experiencias didácticas publicadas hasta el 2018 (Scott y Goode, 2020), se carecen de posibles líneas de investigación educativas o proyecciones ante la actual transformación digital del siglo XXI.

En esta dirección, Fernández-Díaz, Rodríguez-Hoyos y Haya-Salmón (2017) abordan una introducción a las líneas de investigación, destacándose: el análisis de las RSD como medios educativos; el uso académico de estas redes y la percepción estudiantil ante las RSD; sin embargo se centran fundamentalmente en resultados obtenidos en España. En una actualización del tema, Scott y Goode (2020) profundizan en las limitaciones de las RSD y la educación ética, pero asociado fundamentalmente al ámbito de la educación médica. Lo anterior motivó a la realización de este trabajo, determinándose como objetivo: determinar a partir de una revisión sistemática las tendencias actuales del uso de las RSD con fines educativos.

\section{Método}

Se empleó el protocolo PRISMA (Urrútia \& Bonfill, 2010) diseñándose tres fases.

- Fase 1: Extracción de la información científica procedente de revistas y actas de conferencias indexadas en Scopus en el periodo 2015-marzo de 2020. Se identifica el objetivo; se formulan preguntas de la investigación, criterios de inclusión y exclusión y la estrategia informacional.

- Fase 2: Clasificación y agrupación de la información científica determinando regularidades y tendencias educativas.

- Fase 3: Discusión de los resultados obtenidos en talleres a distancia con investigadores del Centro Nacional de Educación a Distancia de Cuba y por último, la redacción del documento.

Método de revisión. Para reducir el sesgo, el estudio fue desarrollado por tres investigadores. La implementación se efectuó de septiembre de 2019 hasta abril de 2020.

Para cumplir el objetivo se determinaron como preguntas de la investigación:

- Pregunta $1(\mathrm{P} 1)$ : ¿Qué tipo de diseños predominan en la literatura científica?

- Pregunta 2 (P2): ¿Cuáles son los pares de variables dependiente-independiente? 
- Pregunta 3 (P3): ¿Cuáles son las tendencias actuales del uso de las RSD con fines educativos?

En la implementación de las fases, se emplearon en Scopus técnicas de búsqueda de información, utilizándose combinaciones de los operadores lógicos AND y OR. La ecuación general de búsqueda fue "redes sociales" AND "educación"; "redes sociales" AND "educación superior"; "social media" AND "education"; "educational use of social networks"; "social networks" AND "high education".

Los criterios de inclusión fueron: publicaciones relacionadas con las RSD con fines educativos; estar redactados en inglés o español; artículos publicados en revistas o actas de conferencias (rigurosamente seleccionadas), ambos indexados en Scopus. En cuanto a los de exclusión: artículos duplicados o cortos (short papers), tutoriales y artículos con diseños pocos descriptivos o cuyos resultados no son argumentados. En la aplicación de la estrategia de búsqueda, se encontraron 256 artículos (población) y al aplicar los criterios de inclusión y exclusión disminuyeron a 79 (muestra), de ellos el 34.17\% están redactados en español y el $65,83 \%$ en inglés (Figura 1.). Como síntesis del método empleado (Figura 2.) se refleja la participación de expertos en la valoración de la estrategia realizada y de los resultados obtenidos.

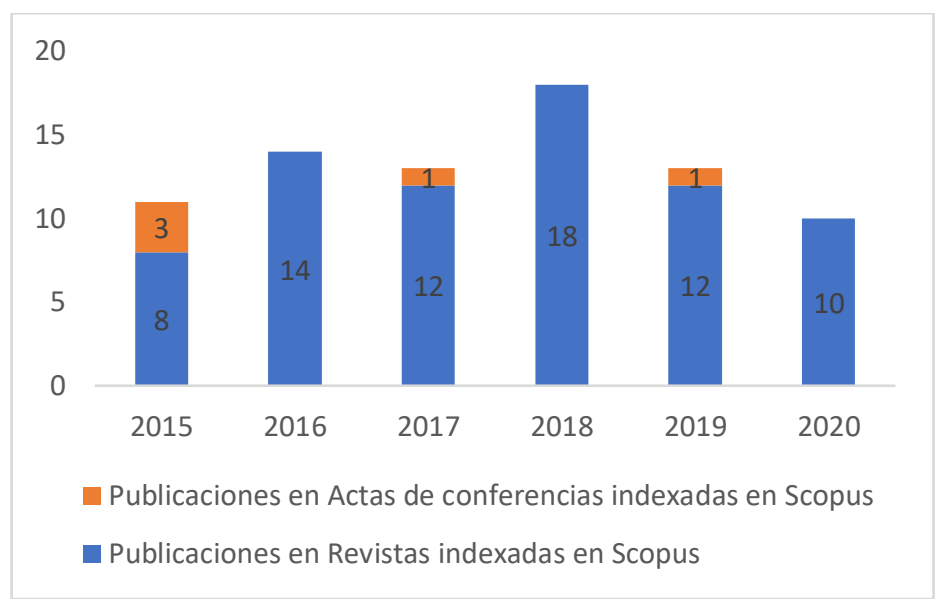

Figura 1. Relación de los 79 artículos revisados

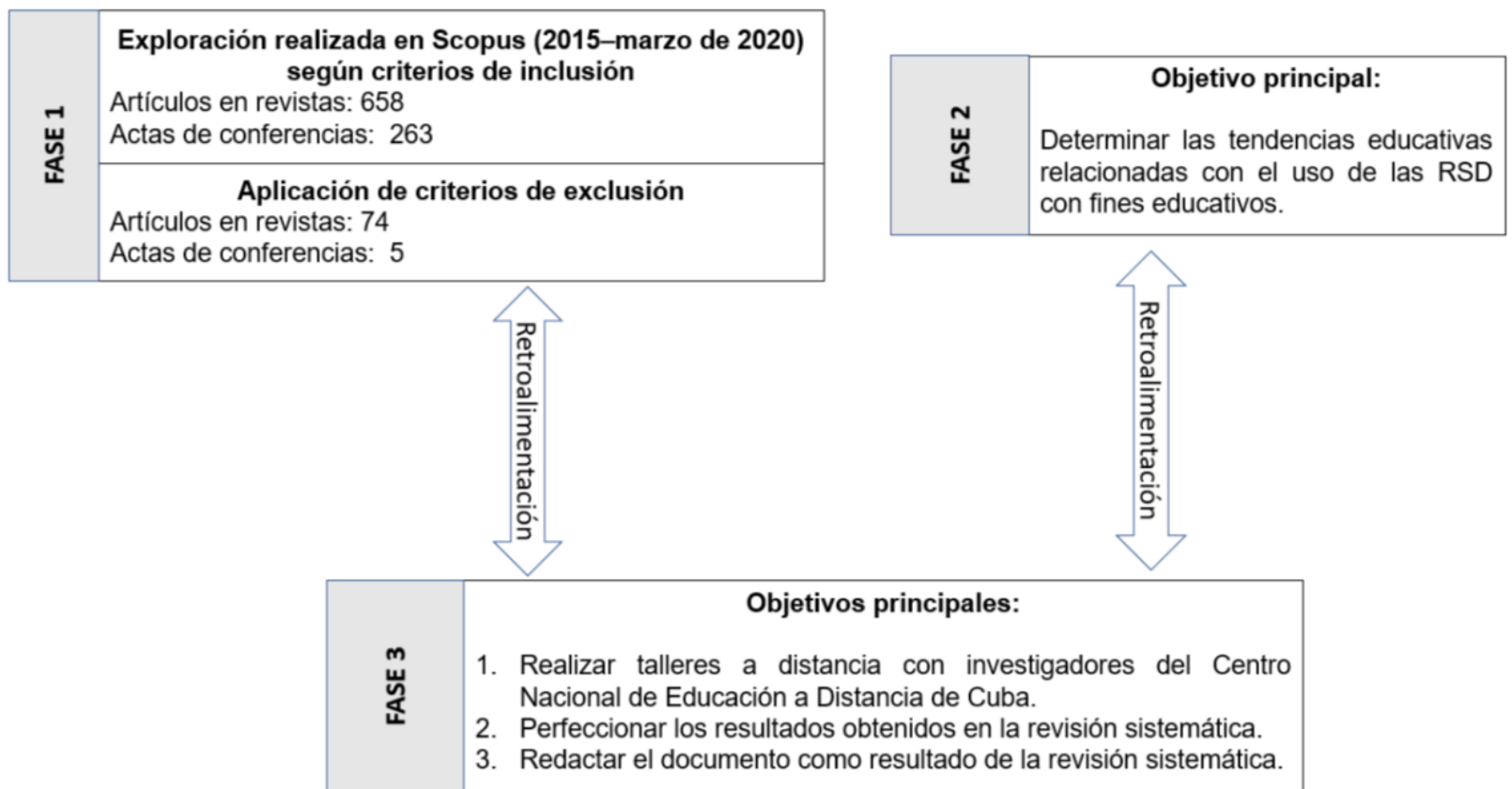

Figura 2. Síntesis de las fases aplicadas en la revisión sistemática

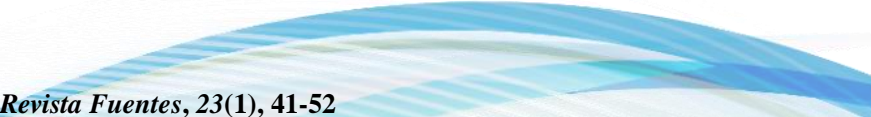


Para disminuir la amenaza de la validez interna y de conclusión se aplicó la técnica Keywording (Odun-Ayo, Goddy-Worlu, Yahaya \& Geteloma, 2019). En cuanto a la validez externa, se descartaron los trabajos con carencias en la argumentación de sus resultados o en su nivel descriptivo. Para lograr la validez del constructo se aplicó el protocolo de revisión abordado anteriormente.

\section{Aportaciones}

Este estudio se sustenta fundamentalmente en una metodología cualitativa. La exposición de los resultados se realiza según las preguntas de la investigación.

En cuanto a la $\mathrm{P} 1$, la revisión permitió identificar que en mayor medida están sustentados en diseños no experimentales ( $n=46$, representando el $58,22 \%$ ), siendo el $41,78 \%$ los experimentales de estos últimos, el $49 \%$ están sustentados en pre experimentos o estudios de casos. (Hernández-Sampieri \& Mendoza, 2018). Las revistas Comunciar; Computers y Education y Píxel-Bit. Revista de Medios y Educación, son las que mayores contribuciones tienen, entre ellas, el $59,25 \%$ son artículos no experimentales y de los experimentales predominan los diseños cuasi experimentales en un $81,81 \%$. (Figura 3 )

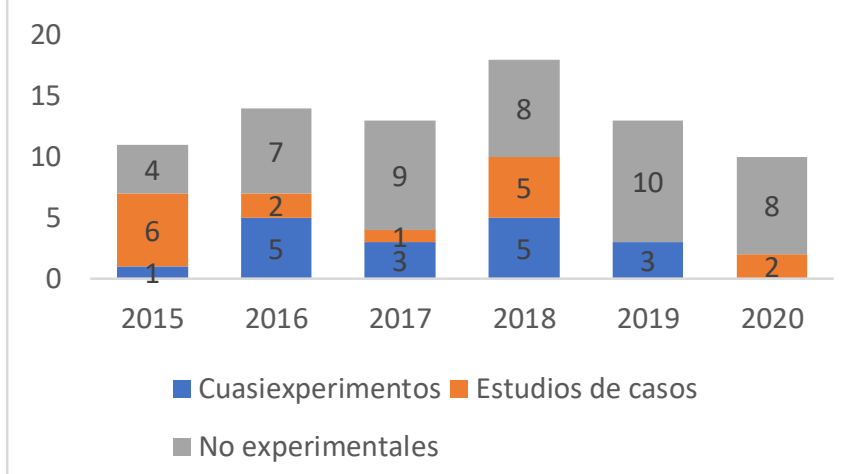

Figura 3. Tipo de diseño presente en las las 79 investigaciones.

Las RSD que más se emplean son Facebook (46,83\%), Twittter (31,64\%), YouTube $(12,65 \%)$, Whatssap y Linkedin (8,86\%) e Instagram (6,32\%) (Figura 4). En estos estudios, en cuanto a la P2, se refleja (Tabla 1) que se ha investigado en disímiles direcciones, siendo 34 variables dependientes, destacándose: e-learning y b-learning (56,96\%); educación formal e informal $(45,56 \%)$; motivación $(53,16 \%)$ e interacción-colaboración $(53,16 \%)$ mientras que la evaluación $(8,86 \%)$ y el rendimiento académico $(6,32 \%)$ fueron los menos trabajados.

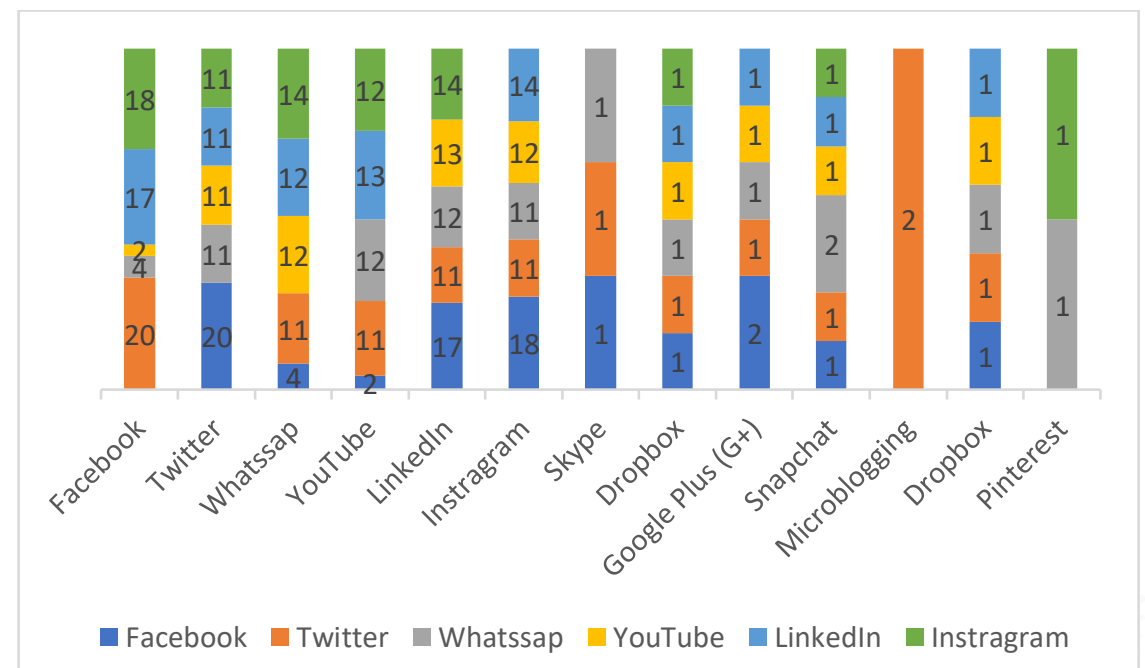

Figura 4. Cantidad de investigaciones según las RDS más estudiadas en las investigaciones. 
En el estudio de las revisiones sistemáticas (Mnkandla \& Minnaar, 2017; Nagle, 2018; Chugh \& Ruhi, 2018; Zachos, Paraskevopoulou-Kollia \& Anagnostopoulos, 2018; Scott \& Goode, 2020), se identificaron como regularidades:

- El uso de las RSD en el aprendizaje electrónico indica que los estudiantes deben ser guiados en la co-construcción del conocimiento y en el empleo integrado de las redes sociales y de los entornos virtuales de enseñanza-aprendizaje (EVEA). En este sentido, es importante potenciar estrategias de aprendizaje social, el compromiso social, la responsabilidad y la pedagogía constructivista.

- Independientemente que no es posible desligar la educación de sus fundamentos filosóficos y sociológicos, el uso de las RDS con fines educativos ha de ser libre de homofilia, ciberviolencia, creencias partidistas, religiosas o ideológicas, potenciando el debate crítico constructivo con personas con diferentes concepciones culturales.

- Promover el empleo correcto del lenguaje, el respeto a la privacidad "digital" del estudiante y del profesor y, la no "invasión pedagógica del profesor" (algunos profesores no logran reconocer las diferencias entre las formas de interactividad e interacción en una RSD al de un EVEA).

Tabla 1. Variables presentes en la revisión bibliográfica

\begin{tabular}{|c|c|c|}
\hline Investigaciones & Principal variable dependiente & $\begin{array}{c}\text { Variable } \\
\text { independiente }\end{array}$ \\
\hline Chang, Guo \& Lin (2017) & Competencia cultural & Actividades en \\
\hline Oberst, Chamarro \& Renau (2016) & Educación de género & Facebook \\
\hline Putnik \& Alves (2019) & Trabajo colaborativo & $\begin{array}{l}\text { Modelo educativo } \\
\text { basado en RSD }\end{array}$ \\
\hline Castaño-Garrido, Maiz-Olazabalaga \& Garay-Ruiz (2015) & Aprendizaje cooperativo & Diseño de de MOOC \\
\hline Del Moral \& Guzmán-Duque (2015) & $\begin{array}{l}\text { Comunidades de aprendizaje } \\
\text { endógenas y exógenas }\end{array}$ & y del uso de RSD \\
\hline Castaño-Garrido, Maiz-Olazabalaga \& Garay-Ruiz (2015a) & $\begin{array}{l}\text { Motivación y rendimiento } \\
\text { académico }\end{array}$ & \\
\hline Hossain, Islam, Glinsky, Lowe, Lowe, Lisa \& Harvey (2015) & E-learning y b-learning & \\
\hline Gil-Quintana \& Martínez-Pérez (2017) & Empoderamiento del alumnado & \\
\hline Delgado-García, García-Prieto \& Gómez Hurtado (2017) & Mediación del aprendizaje & \\
\hline $\begin{array}{l}\text { Marta-Lazo, Osuna-Acedo \& Gil-Quintana (2019); Osuna-Acedo, } \\
\text { Marta-Lazo \& Frau-Meigs (2018) }\end{array}$ & E-learning y b-learning & Diseño de sMOOC \\
\hline Kaplan \& Haenlein (2016) & Educación mediática & $\begin{array}{l}\text { Diseño de MOOCs, } \\
\text { SPOCs y del empleo } \\
\text { de RSD }\end{array}$ \\
\hline Hershkovizt \& Forkosh-Baruch (2017) & Relación alumno-profesor & \multirow{16}{*}{$\begin{array}{l}\text { Estrategias para el uso } \\
\text { de RSD }\end{array}$} \\
\hline Narvaez (2019) & Competencia mediática & \\
\hline Nagle (2018) & $\begin{array}{l}\text { Educación sexual - ciber } \\
\text { violencia }\end{array}$ & \\
\hline Arrabal-Sánchez \& De-Aguilera (2016) & Interacción-Interactividad & \\
\hline Tang \& Hew (2017) & & \\
\hline Alvídrez \& Franco-Rodríguez (2016) & Participación cívica & \\
\hline Logghe, Selby, Boeck, Stamp, Chuen \& Jones (2018) & Rendimiento académico & \\
\hline Oliveira \& Figueira (2015) & Educación abierta & \\
\hline $\begin{array}{l}\text { Eid \& Al-Jabri (2016); Sobaih, Moustafa, Ghandforoush \& Khan } \\
(2016)\end{array}$ & $\begin{array}{l}\text { Motivación y rendimiento } \\
\text { académico }\end{array}$ & \\
\hline $\begin{array}{l}\text { Elitaş (2015); Bicen (2017); Esparza-Puga (2018); Vizcaíno-Verdú, } \\
\text { Contreras-Pulido \& Guzmán-Franco (2019); Hermann-Acosta, } \\
\text { Apolo \& Molano-Camargo (2019); Izquierdo-Iranzo \& Gallardo- } \\
\text { Echenique (2020) }\end{array}$ & Aprendizaje informal & \\
\hline Cabalin \& Antezana (2016). & Orientación ideológica & \\
\hline Denker, Manning, Heuett \& Summers (2018) & $\begin{array}{l}\text { Actitudes, comunicación y } \\
\text { motivación }\end{array}$ & \\
\hline Salas-Rueda (2020) & Aprendizaje automático & \\
\hline Menzies, Petrie \& Zarb (2017) & Aprendizaje colaborativo & \\
\hline Abella \& Delgado (2015) & Aprendizaje informal & \\
\hline González-Pérez (2018) & Autoaprendizaje & \\
\hline
\end{tabular}


Rosón-Jiménez \& Losada-Iglesias (2020)

López-Romero \& Aguaded-Gómez (2015); Grandío-Pérez (2015)

Mayor-Buzón, García-Pérez \& Rebollo-Catalán (2019)

Peña-Hita, Rueda-López \& Pegalajar-Palomino (2018)

Feng, Wong, Wong \& Hossain (2019)

Van Rooyen (2015); Lim \& Richardson (2016); Bunch, Wortman \&

Andriole (2016); Mnkandla \& Minnaar (2017); Rodríguez-Gallego,

López-Martínez \& Martín-Herrera (2017); Canales-García \& Araya-

Muñoz (2017); Picado-Alfaro(2018)

Boude (2016); Borromeo (2016); Fernández-Díaz, Rodríguez-

Hoyos \& Haya-Salmón (2017);Álvarez-Cruces, Otondo-Briceño \&

Medina-Moreno (2018); Moorthy, T'ing, Wei, Zi-Mei, Yee, Jia-Wern

\& Xin (2018); Zachos, Paraskevopoulou-Kollia \& Anagnostopoulos

(2018); Manca (2019); Niu (2019); Thai, Sheeran \& Cummings

(2019); Baker-Doyle \& Yoon (2020); Corona (2020); Scott \& Goode

(2020)

Spanou, Bekiari \& Theocharis (2020)

Gavilan, Martinez-Navarro \& Fernández-Lores (2017); Morón-

Marchena, López-Noguero \& Cobos-Sanchiz (2017)

Lin (2018)

Cascales-Martínez, Gomariz-Vicente \& Paco-Simón (2020)

Willems, Adachi, Bussey, Doherty \& Huijser (2018)

Rodríguez-Gallego,López-Martínez \&Navarro-Montaño (2019)

Valerio y Serna (2018); Awidi, Paynter \&Vujosevic (2019)

Nickerson (2018)

Sharma, Joshi y Sharma (2016); Chugh \& Ruhi (2018); Tubaishat (2018)

Imlawi, Gregg \& Karimi (2015)

Said-Hung, Prati \& Cancino-Borbón (2016)

Sinnema, Daly, Liou \& Rodway (2020)

Castro \& González-Palta (2016)

Saine \& Abraham (2018); Santoveña-Casal \& Bernal-Bravo (2019);

Alaslani \& Alandejani (2020)
Competencia lingüística

Competencia mediática

Competencias digitales

Competencias genéricas

Distracción académica

E-learning y b-learning

Educación mediática

Educación sexual-ciber violencia

Empoderamiento del alumnado

Evaluación

Institución-Familia

Interacción-Interactividad

Método didáctico

Motivación, rendimiento

académico y satisfacción

Orientación ideológica

Patrones de colaboración

Pensamiento crítico

Rendimiento académico

El análisis de la literatura permitió identificar regularidades en las funciones de los siguientes actores:

- Profesor o moderador:

$\checkmark$ Componente cognitivo: dominar competencias mediáticas para lograr (1) diseñar, producir y evaluar recursos educativos digitales y espacios de interacción-colaboración; (2) dirigir la discusión (tipo socio-emocional o centradas en el tema); (3) facilitar y evaluar el aprendizaje estudiantil; (4) diseñar situaciones de aprendizaje basadas en problemas reales y significativos que amplíen la zona de desarrollo próximo; (5) enseñar mediante la orientación pedagógica y del ejemplo profesoral, el cómo emplear las RSD en función del aprendizaje; (6) coordinar la interacción-comunicación desde la diversidad de la comunidad virtual; (7) diagnosticar y caracterizar el aprendizaje de sus estudiantes y sus preferencias en la interacción en redes sociales y, sobre la base de estas, concebir estrategias de enseñanzas.

$\checkmark$ Componente social y actitudinal: Comprender y promover el respeto por la privacidad e intimidad de los estudiantes en las RSD (Vizcaíno-Verdú, Contreras-Pulido \& GuzmánFranco, 2019; Izquierdo-Iranzo \& Gallardo-Echenique, 2020).

- Estudiante:

$\checkmark$ Componente cognitivo: Demostrar el dominio de (1) competencias mediáticas en el empleo de las RSD en un ambiente de participación cívica y colaborativa; (2) competencias lingüísticas y comunicativas para redactar, intercambiar y expresarse correctamente; (3) competencias investigativas para solucionar problemas y actividades de aprendizaje desde una posición científica comunicando sus respuestas de forma sintetizada; (4) competencias tecnológicas para diseñar recursos digitales que le permitan socializar y visualizar los conocimientos adquiridos y (5) competencias de aprender a aprender con y por medio de las RSD. 
$\checkmark$ Componente social, actitudinal y metacognitivo: Comprender e interiorizar la necesidad de la transparencia para la comunicación en las RSD, el compromiso, la autonomía del aprendizaje, la responsabilidad, constancia, el aprendizaje crítico y el respeto. Potenciar una correcta reflexión y regulación metacognitiva (Lin, 2018; Alaslani \& Alandejani, 2020; Spanou, Bekiari \& Theocharis, 2020).

- Comunidad virtual (visto desde la sociología de la educación, no como la suma de integrantes sino como órgano vivo, con identidad propia).

$\checkmark$ Componente cognitivo: Potenciar la mediación social y tecnológica a partir de las experiencias de aprendizaje bajo la dirección pedagógica del profesor. Desarrollar la comunicación asertiva y educativa.

$\checkmark$ Componente social y actitudinal: Comprender que el trabajo grupal y el trabajo individual son elementos de un mismo sistema. Propiciar la contradicción entre el carácter socializador y colectivo de la enseñanza y la naturaleza individual del aprendizaje. (Gil-Quintana \& MartínezPérez, 2017; Rodríguez-Gallego, López-Martínez \& Navarro-Montaño, 2019).

Ahora bien, la transformación digital como parte de la Industria 4.0 exige a la Educación Superior armonizarse a los nuevos tiempos y emplear metodologías para una educación más competitiva y especializada pero matizada según las condiciones sociales, educativas, tecnológicas y laborales de cada sociedad. Para lograr esta meta, el paradigma de la educación (Pedroza 2018; Santaolalla, 2019; Salinas \& Marín, 2019) debe adaptarse con énfasis en la investigación, el desarrollo y la innovación desde la interculturalidad en entornos multiculturales.

Por tal motivo, responder la P3 exige aseverar que el empleo de las RSD con fines educativos también tributa en cierta medida al desarrollo integral y multilateral del estudiante. En esta dirección, a la luz de los estudios analizados y sus variables, se defiende las siguientes tendencias como primordiales en el uso de las RSD con fines educativos:

- Políticas de comunicación institucional universitaria desde los niveles macro, meso y micro del currículo para el uso de las RSD con fines educativos:

Las experiencias didácticas (aisladas) de profesores y grupos de investigación (proyectos de investigación) son vitales para el desarrollo de la educación universitaria, sin embargo, al establecer políticas flexibles propensas a su contextualización en cada asignatura del currículo, contribuirá que el claustro desde sus particularidades cognoscitivas tributen al desarrollo de las competencias digitales, comunicativas y lingüísticas de los estudiantes, estableciéndose para ello pautas para guiar el cómo emplear las RSD desde cada modalidad de estudio.

- Espacios de realización personal:

El empleo de las RSD con fines educativos, debe trascender los límites de los objetivos y contenidos del aprendizaje formal y curricular, potenciando contenidos de aprendizajes abiertos en el que, profesores, estudiantes y la comunidad virtual, sientan satisfacción profesional, personal y motivación en su ambiente de aprendizaje. En esta dirección determinar las dimensiones e indicadores de estos niveles de satisfacción y la relación entre ellos son fundamentales para lograr tal acometida.

- Intercambios de entornos personales de aprendizajes (PLE, por sus siglas en inglés):

La co-construcción del conocimiento a partir del aprendizaje colaborativo y crítico mediado por la comunicación-interacción en las RSD es importante pero también, el cómo el estudiante aprende un contenido determinado y verbaliza su satisfacción con su PLE. Conocer que estrategias empleó, herramientas, bibliografía y recursos, perfeccionará los derroteros del aprendizaje colaborativo, que hoy en día se centra fundamentalmente en el trabajo colaborativo y en la socialización de los resultados alcanzados. La alineación de la pedagogía abierta, colaborativa e inclusiva, es primordial para que el estudiante y profesor comparta esquemas y conocimientos pero también, consejos y experiencias.

- Desarrollo de la creatividad, convergencia cultural y diversificación mediática:

Las situaciones de aprendizaje en las redes sociales deben aprovechar las fortalezas del entretenimiento y las capacidades de visualización, producción compartida, almacenamiento y dinámica social que provee cada red, enriqueciendo las formas de discusión colaborativa y la diversidad de ejercicios más creativos. En esta dirección la diversidad tecnológica, la variedad del aprendizaje y la multiculturalidad son de vital importancia. A su vez, la orientación pedagógica para promover y crear el \#BuenSocialMedia empleando la programación en redes sociales creando contenidos creativos en formato de vídeo, carrusel o stories y logrando que cada profesor y estudiante 
pueda evaluar el desempeño de sus hashtags en Twitter e Instagram, puede ser una alternativa creativa de aprendizaje.

- Cultura mediática para potenciar la relación pensamiento-actitud crítica en el uso de las RSD.

Indistintamente que se necesita profesionales más competentes, profesores y estudiantes deberán a partir del razonamiento crítico en estos escenarios tecnológicos, equilibrar la relación entre: entretenimiento y el aprendizaje formal e informal; la razón y la imaginación; el saber y la información; el arte y la ciencia; educación en las redes sociales y educación con las redes sociales; el aprendizaje colaborativo y significativo con la interacción-comunicación; sentimientos, actitudes y costumbres al respeto y autorregulación a la diversidad cultural; diversidad de situaciones de aprendizaje con la orientación, control y evaluación del aprendizaje y por último, aceptación informacional (de la verdad mediática a partir de la credibilidad y fiabilidad de la información, fake news y del sesgo mediático) y dominio de competencias mediáticas para la búsqueda de la verdad por el método científico en las redes sociales y el Internet en sentido general.

- Innovación educativa:

Los profesores e investigadores deben lograr en las RSD: cambios y originalidad metodológica en la educación mediática y su relación con la actualización tecnológica y las tecnologías emergentes; integrar el aprendizaje formal al informal y lo novedoso con lo tradicional, y por último, evaluar o constatar la durabilidad y fortaleza del aprendizaje en las redes sociales y su relación con el desarrollo de competencias mediáticas, comunicacionales, lingüísticas, investigativas, culturales y profesionales.

- Identidad digital académica:

La educación desde las RSD con fines académicos debe centrarse en cómo se utilizan dichas redes en la práctica social y en el desarrollo de competencias investigativas, digitales y comunicativas, potenciando así un aprendizaje interactivo, colaborativo, informal y abierto al establecimiento de nuevas redes o contactos.

\section{Conclusiones}

El objetivo de esta investigación fue determinar a partir de una revisión sistemática las tendencias actuales del uso de las RSD con fines educativos. En respuesta al objetivo planteado se identificó que estas tendencias son: (1) políticas de comunicación institucional universitaria desde los niveles macro, meso y micro del currículo; (2) espacios de realización personal; (3) intercambios de entornos personales de aprendizajes; (4) desarrollo de la creatividad, convergencia cultural y diversificación mediática; (5) cultura mediática para potenciar la relación pensamiento-actitud crítica en el uso de las RSD y (6) innovación educativa.

Los hallazgos obtenidos coinciden con estudios teóricos que destacan la importancia de la co-construcción del conocimiento y el empleo integrado de las redes sociales y los EVEA (Mnkandla \& Minnaar, 2017; Nagle, 2018; Chugh \& Ruhi, 2018; Zachos, Paraskevopoulou-Kollia \& Anagnostopoulos, 2018). Como sugieren Zachos, Paraskevopoulou-Kollia y Anagnostopoulos, 2018), el empleo de las RSD debe promover espacios de comunicación e interacción colaborativas, libres de ciber violencia, ideas unilaterales partidistas, religiosas, ideológicos y homofóbicas. Por ello, los docentes deben lograr respetar la privacidad digital del estudiante (Scott \& Goode, 2020).

El empleo de las RSD, (Chugh \& Ruhi, 2018; Zachos, Paraskevopoulou-Kollia \& Anagnostopoulos, 2018) implican un reto al profesorado para influir en el rendimiento académico estudiantil, así como en el diseño de ambientes de interacción e interactividad que promuevan el aprendizaje colaborativo y diversifiquen las situaciones de aprendizaje (según los estilos de aprendizaje y la diversidad cultural). A su vez, el desarrollo de competencias investigativas, profesionales y comunicativas son de vital importancia en los últimos ochos años (Scott \& Goode, 2020) potenciando el pensamiento crítico y valorativo.

La diversificación de espacios de interacción-colaboración en los entornos de aprendizaje influyó en la necesidad de aprovechar las funcionalidades de las RSD con énfasis en la mensajería instantánea y la creación, compartición y comentarios a videos y recursos educativos. (Hossain, Islam, Glinsky, Lowe, Lowe, Lisa \& Harvey; 2015; Canales-García \& Araya-Muñoz, 2017; Picado-Alfaro, 2018). En este sentido, se observa una tendencia de emplear las redes en las modalidades del e-learning y b-learning (Marta-Lazo, Osuna-Acedo \& Gil-Quintana, 2019).

Estudios concuerdan en la importancia del empleo de las RSD en la educación formal e informal (EsparzaPuga, 2018; Vizcaíno-Verdú, Contreras-Pulido \& Guzmán-Franco, 2019; Izquierdo-Iranzo \& GallardoEchenique, 2020). En este sentido, se destacan las investigaciones relacionados con el rendimiento 
académico (Logghe, Selby, Boeck, Stamp, Chuen \& Jones, 2018), la orientación ideológica (Cabalin \& Antezana, 2016), el aprendizaje colaborativo (Menzies, Petrie \& Zarb, 2017), las competencias mediáticas, digitales, lingüísticas y genéricas (Grandío-Pérez, 2015; Peña-Hita, Rueda-López \& Pegalajar-Palomino, 2018) y la motivación (Denker, Manning, Heuett \& Summers, 2018; Tubaishat, 2018).

Se reafirma Facebook como la RSD más empleada por los educadores y la necesidad del uso integrado y variado de estas redes, pues los resultados obtenidos coinciden que, por regularidad, las experiencias didácticas presentes en la literatura están basadas en el empleo de una o dos redes sociales lo cual limita la relación entre educación, creatividad, convergencia cultural y las estrategias de aprendizaje.

La investigación ofrece tendencias del uso de las RSD con fines educativos que, de algún modoorientan en dirección a las transformaciones digitales del presente siglo. Se muestran algunos resultados sugerentes relacionados con las variables dependientes-independientes estudiadas en el último quinquenio publicadas en idiomas inglés y español, por lo cual es necesario que la investigación futura explore en otros idiomas para comparar los resultados de la literatura científica.

\section{Referencias}

Abella, V., \& Delgado, V (2015). Learning to use Twitter and use Twitter to learn. Revista de Currículum y Formación del Profesorado, 19 (1), 365 - 378. Recuperado de https://bit.ly/2Xh2VrZ

Alaslani, K., \& Alandejani, M (2020). Identifying factors that influence student's performance through social networking sites: An exploratory case study. Heliyon, 6 (4), e03686. https://doi.org/10.1016/i.heliyon.2020.e03686

Álvarez-Cruces, D., Otondo-Briceño, M., \& Medina-Moreno, A. (2018). Análisis de caso clínico mediante foro virtual por Facebook para favorecer la transferencia de aprendizajes. Educación Médica Superior, 33(2). Recuperado de https://bit.ly/3bEaqOG

Alvídrez, S., \& Franco-Rodríguez, O. (2016). Powerful communication style on Twitter: Effects on credibility and civic participation. Comunicar, 47, 89-97. https://doi.org/10.3916/C47-2016-09

Arrabal-Sánchez, G., \& De-Aguilera, M. (2016). Communicating in 140 characters. How journalists in Spain use Twitter. Comunicar, 46, 9-17. https://doi.org/10.3916/C46-2016-01

Awidi, I. T., Paynter, M., \& Vujosevic, T. (2019). Facebook group in the learning design of a higher education course: An analysis of factors influencing positive learning experience for students. Computers \& Education, 129, 106-121. https://doi.org/10.1016/j.compedu.2018.10.018

Baker-Doyle, K. J., \& Yoon, S. A. (2020). The social side of teacher education: Implications of social network research for the design of professional development. International Journal of Educational Research, 101, 101563. https://doi.org/10.1016/i.ijer.2020.101563

Bicen, $\mathrm{H}$ (2017). Determining the effect of using social media as a MOOC tool. Procedia Computer Science, 120, 172-176, https://doi.org/10.1016/j.procs.2017.11.225

Borromeo, C. (2016). Redes sociales para la enseñanza de idiomas: el caso de los profesores. Píxel-Bit. Revista de Medios y Educación, (48), 41-50. https://doi.org/10.12795/pixelbit.2016.i48.03

Boude, O. (2016). Estrategias de aprendizaje para formar en Educación Superior a una generación interactiva. Educación Médica Superior, 31(2), 1-14. Recuperado de https://bit.ly/3dTgDrC

Bunch, P. M., Wortman, J. R., \& Andriole, K. P. (2016). Social Media and Scientific Meetings: Education Exhibit "Likes" at the Radiological Society of North America Annual Meeting. Academic Radiology, 23(3), 382-388. https://doi.org/10.1016/..acra.2015.10.016

Cabalin, C, \& Antezana, L. (2016). La educación en portada: La visualización de la política educacional en la prensa. Cuadernos.info, (39), 195-207. https://dx.doi.org/10.7764/cdi.39.914

Canales-García, A., \& Araya-Muñoz, I. (2017). Recursos didácticos para el aprendizaje de la educación comercial: Sistematización de una experiencia en educación superior. Revista Electrónica Educare, 21(2), 1-23. https://doi.org/10.15359/ree.21-2.7

Cascales - Martínez, A., Gomariz - Vicente, M., \& Paco - Simón, A. (2020). WhatsApp como herramienta educativa en Educación Primaria: alumnado, docentes y familias.. Píxel-Bit. Revista de Medios y Educación, (58), 71-89. http://dx.doi.org/10.12795/pixelbit.74213

Castaño - Garrido, C., Maiz - Olazabalaga, I., \& Garay - Ruiz, U. (2015). Redes sociales y aprendizaje cooperativo en un MOOC. Revista Complutense de Educación, 26, 119-139. https://doi.org/10.5209/rev RCED.2015.v26.46328

Castaño-Garrido, C., Maiz-Olazabalaga, I., \& Garay-Ruiz, U. (2015a). Design, motivation and performance in a cooperative MOOC course. Comunicar, 44, 19-26. https://doi.org/10.3916/C44-2015-02

Castro, P. J. \& González-Palta, I. N. (2016). Percepción de Estudiantes de Psicología sobre el Uso de Facebook para Desarrollar Pensamiento Crítico. Formación Universitaria, 9(1), 45-56. https://doi.org/10.4067/S071850062016000100006

Chang, L. C., Guo, J. L., \& Lin, H. L. (2017). Cultural competence education for health professionals from pre-graduation to licensure delivered using facebook: Twelve-month follow-up on a randomized control trial. Nurse Education Today, 59, 94-100. https://doi.org/10.1016/i.nedt.2017.09.005 
Chugh, R., \& Ruhi, S. (2018). Social media in higher education: A literature review of Facebook. Education and Information Technologies, 23(2), 605-616. https://doi.org/10.1007/s10639-017-9621-2

Corona, C. (2020). Facebook y otros recursos de la web 2.0 en la enseñanza aprendizaje de la electrocardiografía. Educación Médica Superior, 34(2). Recuperado de https://bit.ly/3fXkPZ2

Del Moral, M. E., \& Guzmán-Duque, A. P. (2015). Comunidades de aprendizaje endógenas y exógenas creadas en torno a los MOOCs universitarios. Campus Virtuales, 4 (2), 78-85. Recuperado de https://bit.ly/3g1OuAD

Delgado-García, M., García-Prieto, F., \& Gómez Hurtado, I. (2017). Moodle y Facebook como herramientas virtuales didácticas de mediación de aprendizajes: opinión de profesores y alumnos universitarios. Revista Complutense de Educación, 29(3), 807-827. https://doi.org/10.5209/RCED.53968

Denker, K. J., Manning, J., Heuett, K. B., \& Summers, M. E. (2018). Twitter in the classroom: Modeling online communication attitudes and student motivations to connect. Computers in Human Behavior, 79, 1-8. https://doi.org/10.1016/i.chb.2017.09.037

Eid, M., \& Al-Jabri, I. M. (2016). Social networking, knowledge sharing, and student learning: The case of university students. Computers \& Education, 99, 14-27. https://doi.org/10.1016/i.compedu.2016.04.007

Esparza - Puga, D. S. (2018). Uso autónomo de recursos de Internet entre estudiantes de ingeniería como fuente de ayuda matemática. Educación matemática, 30(1), 73-91. https://dx.doi.org/10.24844/em3001.03

Feng, S., Wong, Y. K., Wong, L. Y., \& Hossain, L. (2019). The Internet and Facebook usage on academic distractibility and achievement of college students. Computers \& Education, $134,41 \quad-49$. https://doi.org/10.1016/i.compedu.2019.02.005

Fernández-Díaz, E., Rodríguez-Hoyos, C., \& Haya - Salmón, I (2017). Análisis de la investigación nacional e internacional sobre redes sociales en contextos educativos. Revista de Currículum y Formación del Profesorado, 21 (1), 313 - 332. Recuperado de https://bit.ly/2T9mYHd

Gavilan, D., Martinez-Navarro, G., \& Fernández-Lores, S. (2017). University students and informational social networks: Total sceptics, dual moderates or pro-digitals. Comunicar, 53, 61-70. https://doi.org/10.3916/C53-2017-06

Gil - Quintana, J., \& Martínez - Pérez, J. (2017). El empoderamiento del alumnado en los sMOOC. Revista Complutense de Educación, 29(1), 43-60. https://doi.org/10.5209/RCED.51932

González - Pérez, A. (2018). Social networks as tools to enrich learning environments in higher education. Bordón. Revista de Pedagogía, 70(4), 55-71. http://dx.doi.org/10.13042/Bordon.2018.60579

Grandío-Pérez, M. (2015). El transmedia en la enseñanza universitaria. Análisis de las asignaturas de educación mediática en España (2012-2013). Palabra Clave, 19(1), 85-104. Recuperado de https://bit.ly/3dSkWDm.

Hermann-Acosta, A., Apolo, D. E., \& Molano-Camargo, M. (2019). Reflexiones y Perspectivas sobre los Usos de las Redes Sociales en Educación. Un Estudio de Caso en Quito-Ecuador. Información tecnológica, 30(1), $215-224$. https://dx.doi.org/10.4067/S0718-07642019000100215

Hernández-Sampieri, R., \& Mendoza, C. (2018). Metodología de la investigación. Las rutas cuantitativa, cualitativa y mixta. Ciudad de México, México: McGraw-Hill Interamericana de España S.L.

Hershkovizt, A., \& Forkosh-Baruch, A. (2017). Teacher-student relationship and Facebook-mediated communication: Student perceptions. Comunicar, 53, 91-101. https://doi.org/10.3916/C53-2017-09

Hossain, M., Islam, S., Glinsky, J., Lowe, R., Lowe, T., Lisa., \& Harvey, A (2015). A massive open online course (MOOC) can be used to teach physiotherapy students about spinal cord injuries: a randomised trial. Journal of Physiotherapy, 61 (1), 21-27. https://doi.org/10.1016/j.jphys.2014.09.008.

Imlawi, J., Gregg, D., \& Karimi, J. (2015). Student engagement in course-based social networks: The impact of instructor credibility and use of communication. Computers \& Education, 88, 84-96. https://doi.org/10.1016/j.compedu.2015.04.015 Izquierdo-Iranzo, P., \& Gallardo-Echenique, E. (2020). Estudigramers: Influencers del aprendizaje. Comunicar, 62, 115125. https://doi.org/10.3916/C62-2020-10

Kaplan, A., \& Haenlein, M (2016). Higher education and the digital revolution: About MOOCs, SPOCs, social media, and the Cookie Monster. Business Horizons, 59 (4), 441-450. https://doi.org/10.1016/j.bushor.2016.03.008

Lim, J., \& Richardson, J (2016). Exploring the effects of students' social networking experience on social presence and perceptions of using SNSs for educational purposes. The Internet and Higher Education, 29, 31-39. https://doi.org/10.1016/j.iheduc.2015.12.001

Lin, G.Y. (2018). Anonymous versus identified peer assessment via a Facebook-based learning application: Effects on quality of peer feedback, perceived learning, perceived fairness, and attitude toward the system. Computers \& Education, 116, 81 - 92. https://doi.org/10.1016/i.compedu.2017.08.010

Logghe, H. J., Selby, L. V., Boeck, M. A., Stamp, N. L., Chuen, J., \& Jones, C. (2018). The academic tweet: Twitter as a tool to advance academic surgery. Journal of Surgical Research, 226, 8 - 12. https://doi.org/10.1016/i.jss.2018.03.049

López-Romero, L., \& Aguaded-Gómez, M. (2015). Teaching media literacy in colleges of education and communication. Comunicar, 44, 187-195. https://doi.org/10.3916/C44-2015-20

Manca, S. (2019). Snapping, pinning, liking or texting: Investigating social media in higher education beyond Facebook. The Internet and Higher Education, 44, 100707. https://doi.org/10.1016/j.iheduc.2019.100707

Marta-Lazo, C., Osuna-Acedo, S., \& Gil-Quintana, J (2019). sMOOC: A pedagogical model for social inclusion. Helyion, 5 (3), e01326. https://doi.org/10.1016/j.heliyon.2019.e01326 
Mayor - Buzón, V., García - Pérez, R., \& Rebollo - Catalán, Á. (2019). Explorando factores predictores de la competencia digital en las redes sociales virtuales. Píxel-Bit. Revista de Medios y Educación, (56), 51-69. http://dx.doi.org/10.12795/pixelbit.2019.i56.03

Menzies, R., Petrie, K., \& Zarb, M. (2017). A case study of Facebook use: Outlining a multi-layer strategy for higher education. Education and Information Technologies, 22(1), 39-53. https://doi.org/10.1007/s10639-015-9436-y

Mnkandla, E., \& Minnaar, A. (2017). The Use of Social Media in E-Learning: A Metasynthesis. The International Review of Research in Open and Distributed Learning, 18(5). https://doi.org/10.19173/irrodl.v18i5.3014

Moorthy, K., T'ing, L. C., Wei, K. M., Zi Mei, P. T., Yee, C. Y., Jia Wern, K. L., \& Xin, Y. M. (2019). Is facebook useful for learning? A study in private universities in Malaysia. Computers \& Education. https://doi.org/10.1016/i.compedu.2018.12.002

Morón - Marchena, J. A., López - Noguero, F., \& Cobos - Sanchiz, D (2017).El Uso de las Redes Sociales en la Educación y Promoción de la Salud. Una Experiencia de Empoderamiento Social en Nicaragua. Revista de Currículum y Formación del Profesorado, 21 (4), 440 - 457. Recuerado de https://bit.ly/2T6Siai

Nagle, J. (2018). Twitter, cyber-violence, and the need for a critical social media literacy in teacher education: A review of the literature. Teaching and Teacher Education, 76, 86-94. https://doi.org/10.1016/.t.tate.2018.08.014

Narvaez, A. (2019). Comunicación educativa, educomunicación y educación mediática: una propuesta de investigación y formación desde un enfoque culturalista. Palabra Clave, 22(3). https://doi.org/10.5294/pacla.2019.22.3.11

Nickerson, J. P. (2018). Social Media and Radiology Education: Are We \#Ready? Academic Radiology. https://doi.org/10.1016/i.acra.2018.10.015

Niu, L. (2019). Using Facebook for academic purposes: Current literature and directions for future research. Journal of Educational Computing Research, 56(8) 1384-1406. https://doi.org/10.1177/0735633117745161

Núñez, N. (2018). Tenencia, uso y actitudes hacia las TIC: estudio a los directores de primaria y secundaria de centros educativos públicos. En Hacia la sociedad de la información y el conocimiento (pp. 215-236). San José, Costa Rica: Universidad de Costa Rica.

Oberst, U, Chamarro, A., \& Renau, V. (2016). Gender stereotypes 2.0: Self-representations of adolescents on Facebook. Comunicar, 48, 81-90. https://doi.org/10.3916/C48-2016-08

Odun-Ayo, I., Goddy-Worlu, R., Yahaya, J., \& Geteloma, V. (2019). A systematic mapping study of cloud policy languages and programming models. Journal of King Saud University - Computer and Information Sciences. https://dx.doi.org/10.1016/j.jksuci.2019.05.003

Oliveira, L., \& Figueira, Á. (2015). Benchmarking Analysis of Social Media Strategies in the Higher Education Sector. Procedia Computer Science, 64, 779-786. https://doi.org/10.1016/i.procs.2015.08.628

Osuna-Acedo, S., Marta-Lazo, C., \& Frau-Meigs, D. (2018). From sMOOC to tMOOC, learning towards professional transference. ECO European Project. Comunicar, 55, 105-114. https://doi.org/10.3916/C55-2018-10

Pedroza, R. (2018). La Universidad 4.0 con currículo inteligente 1.0 en la cuarta revolución industrial. RIDE Revista Iberoamericana para la Investigación y el Desarrollo Educativo, 9(17), 168-194. https://doi.org/10.23913/ride.v9i17.377

Peña - Hita, M., Rueda - López, E., \& Pegalajar - Palomino, M. (2018). Posibilidades didácticas de las redes sociales en el desarrollo de competencias de Educación Superior: percepciones del alumnado. Píxel-Bit. Revista de Medios y Educación, (53), 239-252. http://dx.doi.org/10.12795/pixelbit.2018.i53.16

Picado-Alfaro, M. (2018). Sugerencias didácticas para la implementación de un curso de nivelación en matemáticas: La sistematización de una experiencia en la Universidad Nacional de Costa Rica. Revista Electrónica Educare, 22(3), 1-18. https://doi.org/10.15359/ree.22-3.15

Putnik, G., \& Alves, C (2019). Learning material co-creation infrastructure in Social Network-based Education: An implementation model. Procedia CIRP, 84, 215-218. https://doi.org/10.1016/i.procir.2019.07.003.

Rodríguez-Gallego, M. R., López-Martínez, A., \& Navarro-Montaño, M. (2019). Longitudinal Study on Social Networks as a Didactic Method. Magis, Revista Internacional de Investigación en educación, 12(24), 85-104. https://doi.org/10.11144/Javeriana.m12-24.Issn

Rodríguez-Gallego, M., López - Martínez, A., \& Martín - Herrera, I. (2017). Percepciones de los estudiantes de Ciencias de la Educación sobre las redes sociales como metodología didáctica. Píxel-Bit. Revista de Medios y Educación, (50), 7793. Recuperado de https://bit.ly/2ZbtfX2

Rosón - Jiménez, M., \& Losada - Iglesias, D. (2020). Microblogging educativo en el aprendizaje de idiomas: una revisión sistematizada de las investigaciones publicadas entre 2010 y 2018. Píxel-Bit. Revista de Medios y Educación, (57), 231251. http://dx.doi.org/10.12795/pixelbit.2019.i57.10

Said-Hung, E., Prati, R., \& Cancino-Borbón, A. (2016). La orientación ideológica de los mensajes publicados en Twitter durante el 24M en España. Palabra Clave, 20(1), 213-238. Recuperado de https://bit.ly/3cE5IS9

Saine, C., \& Abraham, J (2018). Implementing Facebook-based instructional approach in pre-service teacher education: An empirical investigation. Computers \& Education, 18, 1 - 41, doi: https://doi.org/10.1016/i.compedu.2018.09.025

Salas - Rueda, R. (2020). Percepciones de los estudiantes sobre el uso de Facebook y Twitter en el contexto educativo por medio de la ciencia de datos y el aprendizaje automático. Píxel-Bit. Revista de Medios y Educación, (58), 91-115. http://dx.doi.org/10.12795/pixelbit.74056

Salinas, J., \& Marín, V. (2019). Metasíntesis cualitativa sobre colaboración científica e identidad digital académica en redes sociales. RIED. Revista Iberoamericana de Educación a Distancia, 22(2), 97-117. doi:https://doi.org/10.5944/ried.22.2.23238 
Santaolalla, P. (2019). Lograr la equidad en Educación a través de competencias interculturales e intersociales. Revista Fuentes, 21(2), 229-238. https://doi.org/10.12795/revistafuentes.2019.v21.i1.07

Santoveña-Casal, S., \& Bernal-Bravo, C. (2019). Exploring the influence of the teacher: Social participation on Twitter and academic perception. Comunicar, 58, 75-84. https://doi.org/10.3916/C58-2019-07

Scott, N., \& Goode, D. (2020). The use of social media (some) as a learning tool in healthcare education: An integrative review of the literature. Nurse Education Today, 104357. https://doi.org/10.1016/i.nedt.2020.104357

Sharma, S. K., Joshi, A., \& Sharma, H. (2016). A multi-analytical approach to predict the Facebook usage in higher education. Computers in Human Behavior, 55, 340-353. https://doi.org/10.1016/i.chb.2015.09.020

Sinnema, C., Daly, A. J., Liou, Y. H., \& Rodway, J. (2020). Exploring the communities of learning policy in New Zealand using social network analysis: A case study of leadership, expertise, and networks. International Journal of Educational Research, 99, 101492. https://doi.org/10.1016/j.ijer.2019.10.002

Sobaih, A. E., Moustafa, M. A., Ghandforoush, P., \& Khan, M. (2016). To use or not to use? Social media in higher education in developing countries. Computers in Human Behavior, 58, 296-305. https://doi.org/10.1016/i.chb.2016.01.002 Spanou, K., Bekiari, A., \& Theocharis, D. (2020). Bullying y maquiavelismo en la universidad a través del análisis de redes sociales. Revista Internacional de Sociología, 78(1), e151. http://dx.doi.org/10.3989/ris.2020.78.1.18.096

Tang, Y., \& Hew, K. F. (2017). Using Twitter for education: Beneficial or simply a waste of time? Computers \& Education, 106, 97-118. https://doi.org/10.1016/i.compedu.2016.12.004

Thai, M., Sheeran, N., \& Cummings, D. J. (2019). We're all in this together: The impact of Facebook groups on social connectedness and other outcomes in higher education. The Internet and Higher Education, $40,44-49$. https://doi.org/10.1016/j.iheduc.2018.10.001

Tubaishat, A. (2018) Student nurses' perceptions of Facebook as an interactive learning platform in nursing education. Contemporary Nurse, 54(4-5), 456-471. https://doi.org/10.1080/10376178.2018.1530944

Urrútia, G \& Bonfill, X (2010). PRISMA declaration: A proposal to improve the publication of systematic reviews and metaanalyses. Med. Clín, 135, 507-511. https://dx.doi.org/10.1016/j.medcli.2010.01.015

Valerio, A. \& Serna, R (2018). Redes sociales y bienestar psicológico del estudiante universitario. Revista electrónica de investigación educativa, 20(3),19-28. https://doi.org/10.24320/redie.2018.20.3.1796

Van Rooyen, A. (2015). Distance Education Accounting Students' Perceptions of Social Media Integration. Procedia Social and Behavioral Sciences, 176, 444-450. https://doi.org/10.1016/i.sbspro.2015.01.495

Vizcaíno-Verdú, A., Contreras-Pulido, P., \& Guzmán-Franco, M. (2019). Reading and informal learning trends on YouTube: The booktuber. Comunicar, 59, 95-104. https://doi.org/10.3916/C59-2019-09

Willems, J., Adachi, C., Bussey, F., Doherty, I., \& Huijser, H. (2018). Debating the use of social media in higher education in Australasia: Where are we now? Australasian Journal of Educational Technology, 34(5), 135-149. https://doi.org/10.14742/ajet.3843

Zachos, G., Paraskevopoulou-Kollia, E. A., \& Anagnostopoulos, I (2018). Social Media Use in Higher Education: A Review. Education Sciences, 8(4), 194 - 105, https://doi.org/10.3390/educsci8040194 